(2) Open Access Full Text Article

REVIEW

\title{
Exosomes are fingerprints of originating cells: potential biomarkers for ovarian cancer
}

\author{
This article was published in the following Dove Press journal: \\ Research and Reports in Biochemistry \\ 18 March 2015 \\ Number of times this article has been viewed
}

\section{Miharu Kobayashi \\ Gregory E Rice \\ Jorge Tapia \\ Murray D Mitchell \\ Carlos Salomon}

Exosome Biology Laboratory, Centre for Clinical Diagnostics, University of Queensland Centre for Clinical Research, Royal Brisbane and Women's Hospital, Brisbane, QLD, Australia.
Correspondence: Carlos Salomon Exosome Biology Laboratory Head, Centre for Clinical Diagnostics, University of Queensland Centre for Clinical Research, Building 71/918,

Royal Brisbane Hospital, Herston,

QLD 4029, Australia

Tel +6I 733465044

Email c.salomongallo@uq.edu.au

\begin{abstract}
The past decade has seen an extraordinary explosion of research in the field of extracellular vesicles, especially in a specific type of extracellular vesicles originating from endosomal compartments, called exosomes. Exosomes are a specific subtype of secreted vesicles that are defined as small $(\sim 30-120 \mathrm{~nm})$ but very stable membrane vesicles that are released from a wide range of cells, including normal and cancer cells. As the content of exosomes is cell type specific, it is believed that they are a "fingerprint" of the releasing cell and its metabolic status. We hypothesized that the exosomes and their specific exosomal content (eg, microribonucleic acid) represent a precious biomedical tool and may be used as biomarkers for the diagnosis and prognosis of malignant tumors. In addition, exosomes may modify the phenotype of the parent and/or target cell by transferring pro-oncogenic molecules to induce cancerous phenotype of recipient cells and contribute to the formation of the premetastatic niche. The mechanism involved in these phenomena remains unclear; however, inclusion of signaling mediators into exosomes or exosome release may reduce their intracellular bioavailability in the parent cell, thereby altering cell phenotype and their metastatic potential. The aim of this review therefore is to analyze the biogenesis and role of exosomes from tumor cells, focusing primarily on ovarian cancer. Ovarian cancer is the most lethal gynecologic cancer, and an effective early diagnosis has the potential to improve patient survival. Ovarian cancer currently lacks a reliable method for early detection, however, exosomes have received great attention as potential biomarkers and mediators of the disease.
\end{abstract}

Keywords: extracellular vesicles, exosomes, cancer, metastasis

\section{Introduction}

With over 225,000 new cases and 140,000 deaths reported every year worldwide, ovarian cancer is the most lethal malignancy among all gynecologic cancers. ${ }^{1}$ The main contributing factor to the high mortality rate of ovarian cancer is late diagnosis. The field of nanovesicle-mediated cell-to-cell communication is of considerable contemporary interest and a burgeoning field that may provide unique insights into gynecologic cancers' etiology, early detection, and treatment monitoring. In this regard, cell-to-cell communication is essential to assure correct organization between different cell types inside tissues. Recent studies have suggested that cells may also communicate with each other through extracellular vesicles (EVs) as carriers of specific molecules. ${ }^{2,3}$ Cells secrete a wide range of EVs of different size, morphology, content, and function that interact with target cells and modify their phenotype and function. Recent reports have recognized a specific type of nanovesicle, the exosomes, as a tumor "courier" carrying signal and relocating packages of information to initiate metastasis. ${ }^{4,5}$ Exosomes are 
small (30-120 nm) membrane vesicles of endocytic origin that are actively released by tumor cells into the peripheral circulation and play a role in tumor progression and metastasis. Moreover, the concentration of exosomal protein in plasma has been reported to increase in association with disease severity and/or progression in patients with ovarian cancer. $^{6}$

\section{Ovarian cancer}

Ovarian cancer is the sixth most commonly reported cancer and the fifth leading cause of cancer-related deaths, accounting for $5 \%$ of all cancer mortality in developed countries. ${ }^{7}$ Data highlights the significance of this disease in the population and its high mortality rate. Progress has been made in ovarian cancer research, however, few improvements have been achieved in survival rates over the years. ${ }^{8}$ Ovarian cancer continues to be a challenging public health issue. One of the main contributing factors to the high mortality rate is late diagnosis. For patients diagnosed at an early stage of the disease (ie, stage 1), when the tumor is still localized to the ovaries, 5-year survival rate is $90 \%$ with currently available treatments. ${ }^{9}$ Once the tumor has disseminated and progressed to the advanced stages (ie, stage $3 / 4$ ), the 5-year survival rate dramatically decreases to $20 \%$ or less. ${ }^{9}$ Early diagnosis and removal of the tumor therefore have the potential to significantly improve mortality rate. Unfortunately, only $15 \%$ of patients are currently diagnosed when the tumor is still localized to the ovaries. ${ }^{10}$ Reduction in mortality does not solely depend on the development of effective treatment but also on the detection of cancer at an early stage while it is still treatable. Ovarian cancer currently lacks a reliable method for early detection, however, exosomes have received great attention as a potential biomarker and mediator of the disease. Recently, it has been demonstrated that ovarian cancerderived exosomes carry a wide range of specific molecules (ie, proteins) associated with carcinogenesis. ${ }^{11}$ The effects of cancer environmental factors on the mechanisms involved in the exosome biogenesis still remain to be understood.

Despite active research to reduce disease-associated mortality through early detection, there has been limited advancement. Early-stage ovarian cancer is not associated with overt symptoms, and no reliable diagnostic method is available. For many years, ovarian cancer has been portrayed as a "silent killer" due to the lack of early symptoms. ${ }^{12,13}$ Recent studies have reported that $95 \%$ of ovarian cancer patients and $89 \%$ of early-stage ovarian cancer patients experience some symptoms prior to diagnosis. ${ }^{14}$
The reported symptoms were predominantly nongynecologic, such as abdominal pain/swelling, gastrointestinal disturbance, and bloating, while gynecologic symptoms were the least commonly reported. ${ }^{12}$ Since the symptoms tend to be nonspecific and common in the general population, patients are often initially investigated within nongynecologic clinical departments, which often leads to missed or delayed diagnosis. ${ }^{15}$

The treatment of ovarian cancer is challenging once metastasis has occurred. Ovarian cancer differs from the classic model of metastasis. While most types of tumor spread hematogenously, ovarian cancer tumor cells are released into the peritoneal cavity. ${ }^{16}$ Approximately $90 \%$ of ovarian cancer patients are diagnosed with epithelial ovarian cancer (EOC), which can further be classified into serous, mucinous, endometrioid, clear cell, and mixed based on its histology. ${ }^{15,17}$ Serous carcinoma is the most common EOC, accounting for $70 \%$ of all EOC. ${ }^{17}$ Traditionally, it was thought that serous carcinoma originates from epithelial cells lining the ovarian surface or subsurface of inclusion cysts; however, there is emerging evidence that serous carcinoma can arise from the epithelium of the fallopian tube and spill onto the surface of the ovary. ${ }^{18}$

\section{Exosome biogenesis and function}

Cells secrete heterogeneous populations of EVs. Many terms have been used to refer to these small membrane-enclosed vesicles (eg, microvesicles, nanovesicles, shedding vesicles, and ectosomes), emphasizing the wide range of EV populations reported and the difficulty in their determination. ${ }^{19}$ A specific population of EVs, known as exosomes, can be distinguished from others based on their size, morphology, composition, and endosomal origin. ${ }^{19}$ Unlike other EVs secreted by direct budding of the plasma membrane, exosomes are generated by the intraluminal invagination of early endosomes, giving rise to multivesicular bodies (MVBs) or multivesicular endosomes containing intraluminal vesicles (ILVs), and released into the extracellular environment upon the fusion of MVBs/multivesicular endosomes with the plasma membrane (Figure 1). ${ }^{20}$ Exosomes are homogeneous in size compared with other EVs, ranging from $30 \mathrm{~nm}$ to $120 \mathrm{~nm}$ in diameter (Figure 2A and B), which is consistent with the reported in situ size of ILVs. ${ }^{21}$ Due to their endosomal origin, exosomes carry proteins associated with MVB biogenesis (eg, tetraspanins, Alix, and Tsg101) and membrane fusion (eg, Rab GTPase, annexins). ${ }^{19}$ The endosomal sorting complex required for transport (ESCRT) pathway facilitates membrane remodeling (ie, bending or 


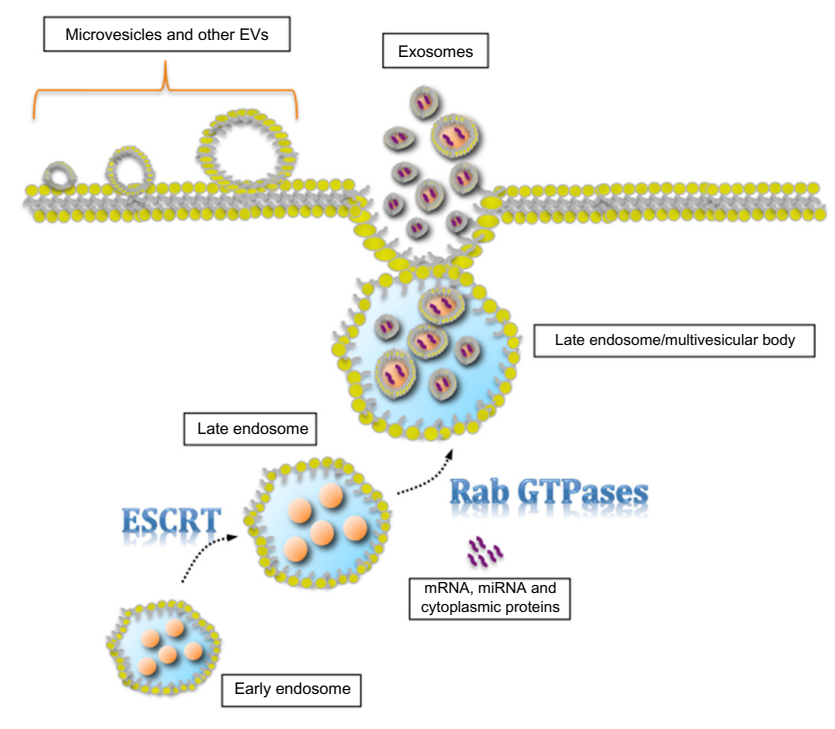

Figure I Schematic diagram of exosome biogenesis and secretion.

Notes: Exosomes are generated in the endosomal structure and secreted via endosomal pathways. The exosomes contain specific proteins and microribonucleic acid, mediating intercellular communication to modify the different biological function of target cells.

Abbreviations: EVs, extracellular vesicles; ESCRT, endosomal sorting complex required for transport.

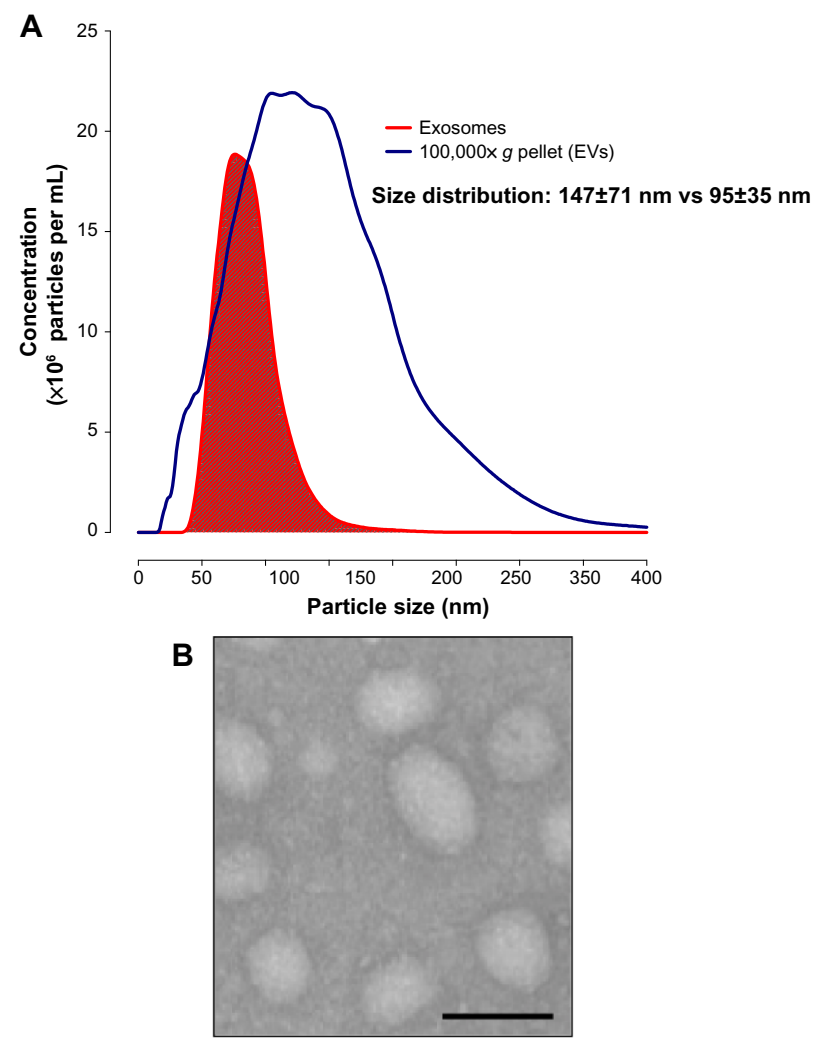

Figure 2 Extracellular vesicle (EV) size distribution and exosome morphology. Notes: (A) Representative vesicle size distribution measured by the nanoparticle tracking analysis NanoSight NS500 instrument. Blue: 100,000× g pellet (EVs); red: enriched exosome population. (B) Representative electron micrograph of exosome population, scale bar $100 \mathrm{~nm}$. budding away from the cytoplasm) and has been implicated in the formation of ILVs. ${ }^{22}$ Evidence suggests that ESCRT-independent pathways may also contribute to exosome formation, as MVBs are formed when all four ESCRT complex subunits are repressed. ${ }^{23,24}$ Mature MVBs are transported to the plasma membrane, where exosomes are released upon the fusion of MVBs with the plasma membrane promoted by Rab GTPases. ${ }^{25,26}$ Exosomes are released from a wide range of cell types and have been isolated from various bodily fluids, including blood, saliva, breast milk, ascites, and amniotic fluids. ${ }^{27-31}$ Initially, exosomes were considered to be a mechanism by which unwanted cell products were eliminated. ${ }^{32}$ Recent studies, however, have provided persuasive data establishing the role of exosomes in cell-to-cell communication. ${ }^{33}$

\section{Exosomes: a new mode of intercellular communication}

Our understanding of the mechanism(s) by which exosomes interact with recipient cells remains to be fully established. The available data are consistent with several possible modes of communication, including: 1) receptor-ligand interaction on cell and exosome surfaces; 2) exosomes attaching or fusing with the cell membrane, directly delivering exosomal surface proteins and cytosol; and 3) internalization of exosomes by recipient cells through phagocytosis. ${ }^{34-37}$ It has been demonstrated that cells internalize exosomes through lipid raft-mediated endocytosis involving caveolin-1 protein and ERK1/2-heat shock protein 27 signaling. ${ }^{38}$ Their molecular cargo is cell specific, regulated by tissue physiology and cellular function and fundamental to their bioactivity. ${ }^{39}$

Cells acquiring tumorigenic traits (ie, unregulated cell proliferation and resistance to cell death) are insufficient for tumor progression. Multiple distinct cell types are involved in the process, requiring effective cell-to-cell communication between cancer cells and local/distant microenvironments. ${ }^{40}$ There has been increasing interest in the role of exosomes as a mediator of cell-to-cell communication between these cells and microenvironments for effective cancer progression.

Tumor-derived exosomes may affect cancer progression in several ways. Tumor-derived exosomes have the ability to propagate oncogenic activity among tumor cells. Human glioma cells can horizontally transfer an oncogenic form of epidermal growth factor variant 3 (EGFRvIII) to glioma cells lacking EGFRvIII. ${ }^{41}$ The transfer results in increased expression of prosurvival genes and reduction of cell cycle inhibitors, increasing the anchorage-independent growth capacity. ${ }^{41}$ 
Exosomes not only facilitate communication among tumor cells but also develop favorable microenvironments for tumor progression. Angiogenesis is promoted by the activation of endothelial cells by tumor-derived exosomes and is supported by the activation of myofibroblasts, a source of matrix-remodeling proteins. ${ }^{42,43}$ Tumor-derived exosomes trigger fibroblast to myofibroblast differentiation. ${ }^{44}$ In addition to fibroblasts, mesenchymal stem cells from tumor stroma and adipose tissue are converted to myofibroblasts through exosomes. ${ }^{45,46}$

Exosomes also contribute to the formation of premetastatic niches by educating bone marrow-derived cells. ${ }^{5}$ Bone marrow-derived cells treated with exosomes derived from highly and poorly metastatic melanoma cells promoted primary tumor growth as well as increased size and number of metastases. ${ }^{5}$ There is increasing evidence that exosomes interact with immune cells to suppress antitumor response and skew toward protumorigenic phenotype. ${ }^{47}$ Tumor cells can interact with different cell types through exosomes, most likely leading to a complex network of interactions.

The effects of exosomes on cell target depend on the exosomal composition by the transfer of a diverse range of bioactive molecules. Exosomes have been reported to carry a diverse range of cell surface receptors, proteins (eg, heat shock proteins, cytoskeletal proteins, adhesion molecules, membrane transport and fusion proteins), and microribonucleic acid (miRNA), with the potential to affect the acute and long-term function of the cells with which they interact. .,48 $^{-48}$

\section{Exosomal miRNAs}

miRNAs are small (about 22 nucleotides in length), noncoding RNAs that regulate gene expressions. ${ }^{49,50}$ They target complementary messenger RNA (mRNA) by binding to their $3^{\prime}$ untranslated region for translational inhibition or degradation. ${ }^{51}$ Many miRNAs are phylogenetically conserved across species, with regulatory impacts on fundamental biological processes such as proliferation, differentiation, and apoptosis. ${ }^{50-52}$ Although miRNA expression is tightly regulated in normal physiology, it is often misregulated in pathophysiology such as cancer.

miRNA profiles can differentiate ovarian cancer tissues from benign tissues, highlighting the diagnostic potential of miRNA. Previous studies have profiled miRNA expression of tumor tissues; however, tissue sample collection is unsuitable for diagnostic and screening purposes, due to its invasive methods. Therefore, miRNAs present in biofluids, such as those carried in exosomes, have attracted interest as cancer biomarkers. Exosomes have been found to mediate
miRNA transfer between cells. ${ }^{53}$ miRNA profile in exosomes is specific to the tumor cells of origin and protected from degradation from molecules such as RNase, which is present in most biofluids.

Recently, we defined the exosomal miRNA expressions of the let-7 and miR-200 families from ovarian cancer cells (SKOV-3 and OVCAR-3 cell lines) by real-time polymerase chain reaction. ${ }^{39}$ The expression of let-7 miRNA transcripts was significantly greater in high invasive SKOV-3 cellderived exosomes compared with exosomes released from low invasive OVCAR-3 cells. miRNA expression of SKOV-3 cell-derived exosomes and their originating cells showed no correlation; however, miRNA profiles were highly correlated between OVCAR-3 cell-derived exosomes and their cells of origin. Exosomal miRNA contents are significantly different between ovarian cancer cell lines, with the profiles correlating to the invasive potential of their originating cells. The difference in correlation between the exosomal and cellular miRNA expression establishes the selective packaging of the miRNA contents into exosomes, suggesting a mechanism to maintain cell invasive phenotype by regulating miRNA transcripts with tumor-suppressing activity, such as the let-7 family in cells. ${ }^{39}$ Vaksman et $\mathrm{al}^{54}$ have demonstrated the potential use of exosomal miRNA as a prognosis marker since miRNA expression in exosomes isolated from ovarian cancer patient peritoneal and pleural effusions correlated with disease outcome. High exosomal miR-21, miR-23b and miR-29a expression of ovarian cancer effusion correlated with progression-free survival, while high expression of miR-21 was associated with poor overall survival..$^{54}$

\section{Ovarian cancer metastasis}

Epithelial to mesenchymal transition (EMT) is one of the most important events taking place during the early phase of ovarian cancer metastasis. EMT is a process in which epithelial cells lose epithelial characteristics such as cell polarity and cell-to-cell junctions, acquiring mesenchymal cell characteristics to gain cell motility and invasiveness. ${ }^{55}$ The acquired mesenchymal properties allow cells to detach from the primary tumor into the peritoneum, where cells often aggregate to form spheroid-like structures. ${ }^{56,57}$ These aggregates are transported throughout the peritoneal cavity by normal peritoneal fluid and implant on the surfaces of the abdominal cavity or the organs therein, forming tumors at a secondary site. ${ }^{16,57}$ Although peritoneal cavity has been believed to be the mode of epithelial ovarian tumor metastasis, a recent study suggests an alternative route, hematogenous metastasis. Ovarian cancer cell injected to 
one of the two surgically attached mice, sharing a common circulation (parabiosis model) metastasized to the omentum of the other mice. ${ }^{58}$

\section{Potential role of exosomes in promoting a protumor environment}

Tumor cells can modulate cellular activity of target cells through the transfer of molecules via exosomes. Exosomes isolated from peritoneal effusions collected from ovarian cancer patients contained low expression of programmed cell death 4 , whereas oncogenic microRNA-21 (miR-21), known to downregulate programmed cell death 4 expression by directly targeting its $3^{\prime}$-untranslational region, was highly expressed compared with those isolated from nonneoplastic peritoneal effusions. ${ }^{59}$ These observations provide an interesting possibility that exosomes are critical factors affecting neighboring cells.

Despite incomplete understanding of the mechanisms underlying exosome release, hypoxia, and thermal and oxidative stress, anticancer drugs are known factors triggering the release of exosomes. ${ }^{60-62}$ Hypoxia, the reduction of oxygen tension, is the key regulatory factor of cancer progression. ${ }^{63}$ Adaptive changes are induced in cancer cells under a hypoxic microenvironment, contributing to their aggressive behavior and poor patient outcomes. ${ }^{64}$ We have extensively quantified the exosome release from placental cells (placental cells share similar characteristics with cancer cells, such as their invasive and migration capacity) under different oxygen tension (ie, 8\%, 3\%, and 1\% $\mathrm{O}_{2}$ ). ${ }^{48,65,66}$ Studies have established an inverse correlation between placental cell exosome release and oxygen tension. Low oxygen tension increased exosome release from umbilical cord-derived mesenchymal stem cells by $\sim 5.6$-fold. ${ }^{67}$ Increased exosome release was observed in cardiac myocytes as well as cancer cell lines: squamous carcinoma cells (A431 cells) and breast cancer cell lines (MCF7, SKBR3, and MDA-MB 231). ${ }^{68,69}$ The underlying mechanism in which hypoxia induces exosome release remains to be fully elucidated; however, evidence suggests the role of transcriptional factor HIF- $1 \alpha$ in hypoxia-induced exosome release.$^{60}$ In the same study, high expression of miR-210 was observed in exosomes released from cancer cells cultured under hypoxic conditions compared with those isolated from normoxic conditions. Endothelial cell angiogenesis is promoted by miR-210 in metastatic cancer cell-derived exosomes. ${ }^{70}$

Genes that promote survival, proliferation, angiogenesis, invasion, and metastatic spread are upregulated under hypoxic conditions supporting growth of a primary tumor microenvironment. For instance, hypoxia induces EMT in pancreatic tumor cells, as evidenced by downregulation of epithelial marker E-cadherin, upregulation of mesenchymal marker vimentin, morphological changes in cells, and increased migration rate. ${ }^{71}$ Similarly, hypoxia shifted the expression of E-cadherin to vimentin at both the mRNA and protein levels and promoted invasiveness and migration in ovarian cancer cells. $^{72,73}$ Exosomes secreted under hypoxia from prostate cancer cells contained proteins associated with remodeling of epithelial adherens junctions and downregulated the expression of E-cadherin at the membrane of target prostate cancer cells. ${ }^{74}$ Human embryonic kidney cells treated with exosomes expressing HIF- $1 \alpha$ can induce expression of EMTassociated markers. ${ }^{75}$

Recently, miRNA, specifically the members of the miR-200 family (miR-200a, miR-200b, miR-200c, miR-141, and miR-429), has attracted great attention as a regulator of EMT in cancer metastasis. Korpal et $\mathrm{al}^{76}$ demonstrated that miR-200 family expression was downregulated over time when EMT was induced in mouse mammary epithelial cells by transforming growth factor beta, and the overexpression of miR-200 family hindered transforming growth factor beta-mediated EMT. Additionally, inhibition of miR-200 family-induced EMT and ectopic expression of the family in mesenchymal cells initiated mesenchymal to epithelial transition. ${ }^{77}$ Overexpression of miR-200 family inhibits EMT by targeting E-cadherin repressors ZEB1 and ZEB2 ${ }^{78}$ The miR-200 family is one of the most deregulated miRNAs in epithelial ovarian tumors compared with normal ovarian tissues..$^{79}$ Aberrantly expressed miR-200 family in ovarian cancer tumor was similarly expressed in originating cells and exosomes released from those cells. ${ }^{6}$ These studies suggest the potential role of exosomes in hypoxia-induced EMT.

\section{Challenges of early-stage ovarian cancer diagnosis}

The absence of adequate early detection strategies is an additional factor contributing to the challenge of early diagnosis. Cancer antigen 125 (CA-125) is currently the most widely used serum biomarker for ovarian cancer. CA-125, however, is approved by the US Food and Drug Administration only to monitor disease treatment and not as a screening test. Although CA-125 is known to be elevated in over $80 \%$ of advanced-stage ovarian cancer patients, elevated CA-125 concentrations are observed in only $50 \%-60 \%$ of early-stage patients, and up to $20 \%$ of ovarian cancer tissues do not express CA-125. ${ }^{9,80}$ Furthermore, CA-125 is not specific to ovarian cancer, as other malignancies, nongynecologic disease, age, menstrual cycle, and pregnancy can influence serum CA-125 
concentrations. ${ }^{81}$ Regardless of its widespread off-label use, CA-125 alone is not sufficiently sensitive or specific for early detection. Currently, investigation and disease confirmation in symptomatic women therefore rely on a combination of transvaginal ultrasound and monitoring CA-125 serum concentrations over time. Transvaginal ultrasound defines ovarian morphology as well as any morphological changes that may signify malignancy development. ${ }^{81,82}$ This method can result in high false-positive rates, as it is difficult to distinguish benign abnormalities from malignancy, thus resulting in unnecessary surgical intervention. ${ }^{80,82}$

As currently available biomarkers and screening techniques have failed to improve clinical outcomes, the development of early detection strategies for ovarian cancer is essential. Ideal approaches should involve minimally invasive procedures with greater sensitivity. The recent explosion of exosome research has strongly emphasized the application of these nanovesicles as diagnostic and treatment monitoring tools; however, extensive studies to characterize the content, role, release of exosomes, and their utility as biomarkers for cancer, including ovarian cancer, have yet to be performed..$^{83,84}$

\section{Exosomes as a source of cancer biomarkers}

The following features of exosomes make them an ideal source of cancer biomarkers: 1) actively released by live tumor cells, 2) content reflects the tumor state and its microenvironment, 3 ) easily collected from a wide range of biofluids, 4) easily isolated from high-abundance proteins present in biofluids confounding biomarker discovery, and 5) highly stable. These exosomes are released from living tumor cells and can be differentiated from microvesicles secreted from apoptotic cells. ${ }^{85}$ The presence of specific cellular protein and RNA from originating cells provides comprehensive information about the tumor.

Exosomes provide an enriched source of biomarkers. They can be collected through minimally invasive procedures from the blood or through noninvasive procedures from biofluids such as the urine and saliva. Studies show promising results of exosomes as cancer biomarkers due to their significant correlation with disease stage and their high stability, as storage does not significantly affect their contents. ${ }^{86}$ Exosomes were characterized from the EOC cell lines with different invasive potentials cultured in exosomefree media by the expression of CD63 and CD9 markers with density between $1.15 \mathrm{~g} / \mathrm{mL}$ and $1.19 \mathrm{~g} / \mathrm{mL}$. Exosome release from SKOV-3 cells was greater than that from OVCAR-3 cells by more than twofold when both cells were cultured under identical conditions. The exosome release was specific to the cell type, and their contents correlated to the invasive potential of originating cells. ${ }^{39}$

\section{Conclusions and perspectives}

Despite extensive ovarian cancer research, the mortality rate remains high, with less than half the patients $(44.6 \%)$ surviving for 5 years after diagnosis. ${ }^{10}$ This is mainly due to the incomplete understanding of the molecular mechanism underlying ovarian cancer progression and the lack of reliable diagnostic markers for early detection. Characterizing the content and functions of tumor-derived exosomes has the potential to overcome the current challenges of improving ovarian cancer patient outcomes. The release of exosomes and their molecular cargo is cell type specific, reflecting the state of the originating cells as well as their microenvironment. In other words, exosomes can be considered as a noninvasive "biopsy" of tumor mass, as exosomes can be obtained from a wide range of biological fluids such as blood, urine, and saliva. Not only will exosomes give some insight into the tumor cells themselves but they will provide a better understanding of how other cell types contribute to cancer progression. Cancer is a complex disease involving a wide range of cell types, and effective cell-to-cell communication is essential for successful cancer progression. Exosomes play a role in cell-to-cell communication, and examining the targets and their functions will help identify other cells involved in cancer progression. Initial studies to characterize the protein and miRNA content of exosomes afford some insight into possible mechanisms by which exosomes may affect cell function.

Another challenge of improving ovarian cancer mortality is the development of reliable diagnostic methods. Ovarian cancer lacks a specific symptom, making it difficult to diagnose, however, detectable changes should be taking place at the cellular level. As exosome release is highly dependent on their state and their microenvironment, their content is an ideal biomarker for early detection. As such, the isolation and profiling of exosomes in biological fluids represent a promising approach for the development of detection and monitoring tests; however, the complexity will increase when handling patient samples, as exosomes contained within them can originate from different cell types. Im et al ${ }^{87}$ have recently shown that ovarian cancer-derived exosomes can be identified by the expression of CD24 and EpCAM, suggesting the use of exosomes as a biomarker.

Evidence suggests that vesicles released from cancer cells modify the phenotype of parent and/or target cell by transferring pro-oncogenic molecules, inducing cancerous 
phenotype of receipt cells contributing to tumor growth and metastasis. Exosomes from ovarian carcinoma cells are present in peripheral circulation to enhance tumor development and maintain a favorable environment for cancer progression. Condition-specific changes in the concentration of tumor-derived exosomes may be of clinical utility in the early identification of women with ovarian cancer. Additional experimental studies of ovarian cancer cell-derived exosomes are necessary to further understand ovarian cancer progression, as well as to develop a reliable early biomarker for early diagnosis of patients while the disease is still treatable.

\section{Disclosure}

The authors report no conflicts of interest in this work.

\section{References}

1. Jemal A, Bray F, Center MM, Ferlay J, Ward E, Forman D. Global cancer statistics. CA Cancer J Clin. 2011;61(2):69-90.

2. Zocco D, Ferruzzi P, Cappello F, Kuo WP, Fais S. Extracellular vesicles as shuttles of tumor biomarkers and anti-tumor drugs. Front Oncol. 2014;4:267.

3. Tetta C, Ghigo E, Silengo L, Deregibus MC, Camussi G. Extracellular vesicles as an emerging mechanism of cell-to-cell communication. Endocrine. 2013;44(1):11-19.

4. Brinton LT, Sloane HS, Kester M, Kelly KA. Formation and role of exosomes in cancer. Cell Mol Life Sci. Epub October 22, 2014.

5. Peinado H, Aleckovic M, Lavotshkin S, et al. Melanoma exosomes educate bone marrow progenitor cells toward a pro-metastatic phenotype through MET. Nat Med. 2012;18(6):883-891.

6. Taylor DD, Gercel-Taylor C. MicroRNA signatures of tumor-derived exosomes as diagnostic biomarkers of ovarian cancer. Gynecol Oncol. 2008;110(1):13-21.

7. Ferlay J, Soerjomataram I, Dikshit R, et al. Cancer incidence and mortality worldwide: sources, methods and major patterns in GLOBOCAN 2012. Int J Cancer. 2015;136(5):E359-E386.

8. Siegel R, Naishadham D, Jemal A. Cancer statistics, 2012. CA Cancer J Clin. 2012;62(1):10-29.

9. Bast RC Jr, Badgwell D, Lu Z, et al. New tumor markers: CA125 and beyond. Int J Gynecol Cancer. 2005;15 Suppl 3:274-281.

10. Howlader N, Noone AM, Krapcho M, et al. SEER Cancer Statistics Review 1975-2011. National Cancer Institute. 2014. Available from: http://seer.cancer.gov/csr/1975_2011/. Accessed January 15, 2015.

11. Liang B, Peng P, Chen S, et al. Characterization and proteomic analysis of ovarian cancer-derived exosomes. J Proteomics. 2013;80:171-182.

12. Goff B. Symptoms associated with ovarian cancer. Clin Obstet Gynecol. 2012;55(1):36-42.

13. Smith LH. Early clinical detection of ovarian cancer: a review of the evidence. Expert Rev Anticancer Ther. 2006;6(7):1045-1052.

14. Goff BA, Mandel LS, Drescher CW, et al. Development of an ovarian cancer symptom index: possibilities for earlier detection. Cancer. 2007;109(2):221-227.

15. Lengyel E. Ovarian cancer development and metastasis. Am J Pathol. 2010;177(3):1053-1064.

16. Naora H, Montell DJ. Ovarian cancer metastasis: integrating insights from disparate model organisms. Nat Rev Cancer. 2005;5(5):355-366.

17. McCluggage WG. Morphological subtypes of ovarian carcinoma: a review with emphasis on new developments and pathogenesis Pathology. 2011;43(5):420-432.

18. Erickson BK, Conner MG, Landen CN Jr. The role of the fallopian tube in the origin of ovarian cancer. Am J Obstet Gynecol. 2013;209(5): 409-414.
19. Thery C, Zitvogel L, Amigorena S. Exosomes: composition, biogenesis and function. Nat Rev Immunol. 2002;2(8):569-579.

20. Johnstone RM, Adam M, Hammond JR, Orr L, Turbide C. Vesicle formation during reticulocyte maturation. Association of plasma membrane activities with released vesicles (exosomes). $J$ Biol Chem. 1987;262(19):9412-9420.

21. van Niel G, Porto-Carreiro I, Simoes S, Raposo G. Exosomes: a common pathway for a specialized function. J Biochem. 2006;140(1): 13-21.

22. Colombo M, Moita C, van Niel G, et al. Analysis of ESCRT functions in exosome biogenesis, composition and secretion highlights the heterogeneity of extracellular vesicles. J Cell Sci. 2013;126(Pt 24): $5553-5565$.

23. Stuffers S, Sem Wegner C, Stenmark H, Brech A. Multivesicular endosome biogenesis in the absence of ESCRTs. Traffic. 2009;10(7): 925-937.

24. Trajkovic K, Hsu C, Chiantia S, et al. Ceramide triggers budding of exosome vesicles into multivesicular endosomes. Science. 2008; 319(5867):1244-1247.

25. Savina A, Vidal M, Colombo MI. The exosome pathway in K562 cells is regulated by Rab11. J Cell Sci. 2002;115(Pt 12):2505-2515.

26. Ostrowski M, Carmo NB, Krumeich S, et al. Rab27a and Rab27b control different steps of the exosome secretion pathway. Nat Cell Biol. 2010;12(1):19-30; Suppl 11-13.

27. Runz S, Keller S, Rupp C, et al. Malignant ascites-derived exosomes of ovarian carcinoma patients contain CD24 and EpCAM. Gynecol Oncol. 2007;107(3):563-571.

28. Li J, Sherman-Baust CA, Tsai-Turton M, Bristow RE, Roden RB, Morin PJ. Claudin-containing exosomes in the peripheral circulation of women with ovarian cancer. BMC Cancer. 2009;9:244.

29. Lasser C, Alikhani VS, Ekstrom K, et al. Human saliva, plasma and breast milk exosomes contain RNA: uptake by macrophages. $J$ Transl Med. 2011;9:9.

30. Cheng L, Sun X, Scicluna BJ, Coleman BM, Hill AF. Characterization and deep sequencing analysis of exosomal and non-exosomal miRNA in human urine. Kidney Int. 2014;86(2):433-444.

31. Keller S, Ridinger J, Rupp AK, Janssen JW, Altevogt P. Body fluid derived exosomes as a novel template for clinical diagnostics. J Transl Med. 2011;9:86

32. Pan BT, Johnstone RM. Fate of the transferrin receptor during maturation of sheep reticulocytes in vitro: selective externalization of the receptor. Cell. 1983;33(3):967-978.

33. Futter CE, Pearse A, Hewlett LJ, Hopkins CR. Multivesicular endosomes containing internalized EGF-EGF receptor complexes mature and then fuse directly with lysosomes. J Cell Biol. 1996;132(6): 1011-1023.

34. Raposo G, Nijman HW, Stoorvogel W, et al. B lymphocytes secrete antigen-presenting vesicles. J Exp Med. 1996;183(3):1161-1172.

35. Denzer K, van Eijk M, Kleijmeer MJ, Jakobson E, de Groot C, Geuze HJ. Follicular dendritic cells carry MHC class II-expressing microvesicles at their surface. J Immunol. 2000;165(3):1259-1265.

36. Clayton A, Turkes A, Dewitt S, Steadman R, Mason MD, Hallett MB. Adhesion and signaling by $\mathrm{B}$ cell-derived exosomes: the role of integrins. FASEB J. 2004;18(9):977-979.

37. Morelli AE, Larregina AT, Shufesky WJ, et al. Endocytosis, intracellular sorting, and processing of exosomes by dendritic cells. Blood. 2004;104(10):3257-3266.

38. Svensson KJ, Christianson HC, Wittrup A, et al. Exosome uptake depends on ERK1/2-heat shock protein 27 signaling and lipid Raftmediated endocytosis negatively regulated by caveolin-1.J Biol Chem. 2013;288(24):17713-17724

39. Kobayashi M, Salomon C, Tapia J, Illanes SE, Mitchell MD, Rice GE. Ovarian cancer cell invasiveness is associated with discordant exosomal sequestration of Let-7 miRNA and miR-200. J Transl Med. 2014;12:4.

40. Hanahan D, Weinberg RA. Hallmarks of cancer: the next generation. Cell. 2011;144(5):646-674. 
41. Al-Nedawi K, Meehan B, Micallef J, et al. Intercellular transfer of the oncogenic receptor EGFRvIII by microvesicles derived from tumour cells. Nat Cell Biol. 2008;10(5):619-624.

42. Vong S, Kalluri R. The role of stromal myofibroblast and extracellular matrix in tumor angiogenesis. Genes Cancer. 2011;2(12):1139-1145.

43. Zhuang G, Wu X, Jiang Z, et al. Tumour-secreted miR-9 promotes endothelial cell migration and angiogenesis by activating the JAK-STAT pathway. EMBO J. 2012;31(17):3513-3523.

44. Webber J, Steadman R, Mason MD, Tabi Z, Clayton A. Cancer exosomes trigger fibroblast to myofibroblast differentiation. Cancer Res. 2010;70(23):9621-9630.

45. Cho JA, Park H, Lim EH, et al. Exosomes from ovarian cancer cells induce adipose tissue-derived mesenchymal stem cells to acquire the physical and functional characteristics of tumor-supporting myofibroblasts. Gynecol Oncol. 2011;123(2):379-386.

46. Cho JA, Park H, Lim EH, Lee KW. Exosomes from breast cancer cells can convert adipose tissue-derived mesenchymal stem cells into myofibroblast-like cells. Int J Oncol. 2012;40(1):130-138.

47. Filipazzi P, Burdek M, Villa A, Rivoltini L, Huber V. Recent advances on the role of tumor exosomes in immunosuppression and disease progression. Semin Cancer Biol. 2012;22(4):342-349.

48. Salomon C, Ryan J, Sobrevia L, et al. Exosomal signaling during hypoxia mediates microvascular endothelial cell migration and vasculogenesis. PloS One. 2013;8(7):e68451.

49. Ambros V. The functions of animal microRNAs. Nature. 2004; 431(7006):350-355.

50. Paranjape T, Slack FJ, Weidhaas JB. MicroRNAs: tools for cancer diagnostics. Gut. 2009;58(11):1546-1554.

51. Bartel DP. MicroRNAs: genomics, biogenesis, mechanism, and function. Cell. 2004;116(2):281-297.

52. Miska EA. How microRNAs control cell division, differentiation and death. Curr Opin Genet Dev. 2005;15(5):563-568.

53. Valadi H, Ekstrom K, Bossios A, Sjostrand M, Lee JJ, Lotvall JO. Exosome-mediated transfer of mRNAs and microRNAs is a novel mechanism of genetic exchange between cells. Nat Cell Biol. 2007;9(6): 654-659.

54. Vaksman O, Trope C, Davidson B, Reich R. Exosome-derived miRNAs and ovarian carcinoma progression. Carcinogenesis. 2014;35(9): 2113-2120.

55. Koutsaki M, Spandidos DA, Zaravinos A. Epithelial-mesenchymal transition-associated miRNAs in ovarian carcinoma, with highlight on the miR-200 family: prognostic value and prospective role in ovarian cancer therapeutics. Cancer Lett. 2014;351(2):173-181.

56. Thiery JP. Epithelial-mesenchymal transitions in tumour progression. Nat Rev Cancer. 2002;2(6):442-454.

57. Ahmed N, Thompson EW, Quinn MA. Epithelial-mesenchymal interconversions in normal ovarian surface epithelium and ovarian carcinomas: an exception to the norm. $J$ Cell Physiol. 2007;213(3):581-588.

58. Pradeep S, Kim SW, Wu SY, et al. Hematogenous metastasis of ovarian cancer: rethinking mode of spread. Cancer Cell. 2014;26(1):77-91.

59. Cappellesso R, Tinazzi A, Giurici T, et al. Programmed cell death 4 and microRNA 21 inverse expression is maintained in cells and exosomes from ovarian serous carcinoma effusions. Cancer Cytopathol. 2014;122(9):685-693.

60. King HW, Michael MZ, Gleadle JM. Hypoxic enhancement of exosome release by breast cancer cells. BMC Cancer. 2012;12:421.

61. Hedlund M, Nagaeva O, Kargl D, Baranov V, Mincheva-Nilsson L. Thermal- and oxidative stress causes enhanced release of NKG2D ligand-bearing immunosuppressive exosomes in leukemia/lymphoma $\mathrm{T}$ and B cells. PloS One. 2011;6(2):e16899.

62. Lv LH, Wan YL, Lin Y, et al. Anticancer drugs cause release of exosomes with heat shock proteins from human hepatocellular carcinoma cells that elicit effective natural killer cell antitumor responses in vitro. $J$ Biol Chem. 2012;287(19):15874-15885.

63. Harris AL. Hypoxia: a key regulatory factor in tumour growth. Nat Rev Cancer. 2002;2(1):38-47.
64. Vaupel P, Mayer A. Hypoxia in cancer: significance and impact on clinical outcome. Cancer Metastasis Rev. 2007;26(2):225-239.

65. Ferretti C, Bruni L, Dangles-Marie V, Pecking AP, Bellet D. Molecular circuits shared by placental and cancer cells, and their implications in the proliferative, invasive and migratory capacities of trophoblasts. Hum Reprod Update. 2007;13(2):121-141.

66. Salomon C, Kobayashi M, Ashman K, Sobrevia L, Mitchell MD, Rice GE. Hypoxia-induced changes in the bioactivity of cytotrophoblastderived exosomes. PloS One. 2013;8(11):e79636.

67. Zhang HC, Liu XB, Huang S, et al. Microvesicles derived from human umbilical cord mesenchymal stem cells stimulated by hypoxia promote angiogenesis both in vitro and in vivo. Stem Cells Dev. 2012;21(18): 3289-3297.

68. Byeon YE, Ryu HH, Park SS, et al. Paracrine effect of canine allogenic umbilical cord blood-derived mesenchymal stromal cells mixed with beta-tricalcium phosphate on bone regeneration in ectopic implantations. Cytotherapy. 2010;12(5):626-636.

69. Gupta N, Su X, Popov B, Lee JW, Serikov V, Matthay MA. Intrapulmonary delivery of bone marrow-derived mesenchymal stem cells improves survival and attenuates endotoxin-induced acute lung injury in mice. J Immunol. 2007;179(3):1855-1863.

70. Kosaka N, Iguchi H, Hagiwara K, Yoshioka Y, Takeshita F, Ochiya T. Neutral sphingomyelinase 2 (nSMase2)-dependent exosomal transfer of angiogenic microRNAs regulate cancer cell metastasis. J Biol Chem. 2013;288(15):10849-10859.

71. Salnikov AV, Liu L, Platen M, et al. Hypoxia induces EMT in low and highly aggressive pancreatic tumor cells but only cells with cancer stem cell characteristics acquire pronounced migratory potential. PloS One. 2012;7(9):e46391.

72. Du J, Sun B, Zhao X, et al. Hypoxia promotes vasculogenic mimicry formation by inducing epithelial-mesenchymal transition in ovarian carcinoma. Gynecol Oncol. 2014;133(3):575-583.

73. Imai $\mathrm{T}$, Horiuchi $\mathrm{A}$, Wang $\mathrm{C}$, et al. Hypoxia attenuates the expression of E-cadherin via up-regulation of SNAIL in ovarian carcinoma cells. Am J Pathol. 2003;163(4):1437-1447.

74. Ramteke A, Ting H, Agarwal C, et al. Exosomes secreted under hypoxia enhance invasiveness and stemness of prostate cancer cells by targeting adherens junction molecules. Mol Carcinog. Epub December 17, 2013.

75. Aga M, Bentz GL, Raffa S, et al. Exosomal HIF1alpha supports invasive potential of nasopharyngeal carcinoma-associated LMP1-positive exosomes. Oncogene. 2014;33(37):4613-4622.

76. Korpal M, Lee ES, Hu G, Kang Y. The miR-200 family inhibits epithelial-mesenchymal transition and cancer cell migration by direct targeting of E-cadherin transcriptional repressors ZEB1 and ZEB2. J Biol Chem. 2008;283(22):14910-14914.

77. Gregory PA, Bert AG, Paterson EL, et al. The miR-200 family and miR-205 regulate epithelial to mesenchymal transition by targeting ZEB1 and SIP1. Nat Cell Biol. 2008;10(5):593-601.

78. Park SM, Gaur AB, Lengyel E, Peter ME. The miR-200 family determines the epithelial phenotype of cancer cells by targeting the E-cadherin repressors ZEB1 and ZEB2. Genes Dev. 2008;22(7):894-907.

79. Iorio MV, Visone R, Di Leva G, et al. MicroRNA signatures in human ovarian cancer. Cancer Res. 2007;67(18):8699-8707.

80. Bast RC Jr. Early detection of ovarian cancer: new technologies in pursuit of a disease that is neither common nor rare. Trans Am Clin Climatol Assoc. 2004;115:233-247; discussion 247-248.

81. Gentry-Maharaj A, Menon U. Screening for ovarian cancer in the general population. Best Pract Res Clin Obstet Gynaecol. 2012;26(2): 243-256.

82. Nossov V, Amneus M, Su F, et al. The early detection of ovarian cancer: from traditional methods to proteomics. Can we really do better than serum CA-125? Am J Obstet Gynecol. 2008;199(3):215-223.

83. Taylor DD, Gercel-Taylor C. Tumour-derived exosomes and their role in cancer-associated T-cell signalling defects. Br J Cancer. 2005; 92(2):305-311.

84. Simpson RJ, Jensen SS, Lim JW. Proteomic profiling of exosomes: current perspectives. Proteomics. 2008;8(19):4083-4099. 
85. Thery C, Boussac M, Veron P, et al. Proteomic analysis of dendritic cell-derived exosomes: a secreted subcellular compartment distinct from apoptotic vesicles. J Immunol. 2001;166(12):7309-7318.

86. Sarker S, Scholz-Romero K, Perez A, et al. Placenta-derived exosomes continuously increase in maternal circulation over the first trimester of pregnancy. J Transl Med. 2014;12:204.
87. Im H, Shao H, Park YI, et al. Label-free detection and molecular profiling of exosomes with a nano-plasmonic sensor. Nat Biotechnol. 2014;32(5):490-495.

Research and Reports in Biochemistry

\section{Publish your work in this journal}

Research and Reports in Biochemistry is an international, peer-reviewed open access journal publishing original research, reports, reviews and commentaries on all areas of biochemistry. The manuscript management system is completely online and includes a very quick and fair peer-review system. Visit http://www.dovepress.com/testimonials.php to read real quotes from published authors. 